Eigenkapitalquoten wurden befürchtet (153). Schließlich wurden Zugeständnisse ausgehandelt, aber die Pflicht zur Bewertung der Risiken dennoch eingeführt und die Stellung der Agenturen bestätigt.

Die folgende Finanzkrise thematisiert der Autor erst am Schluss. Dabei werden nur die Maßnahmen angeführt, die US-Börsenaufsicht und EU-Kommission bis 2009 vor allem in technischer Hinsicht vorgenommen haben (158). Eine Kritik der Ratingpraxis bleibt Desiderat. Was Kruck aufgrund des Erscheinungsdatums nicht analysieren konnte und daher annonciert (163ff.), das sind die Reaktionen der EUROStaaten auf die Folgen der anschließenden Bankenrettungsprogramme. Das Narrativ der „Staatsschuldenkrise“ hat sich durchgesetzt. In diesem Kontext ist die Bedeutung von Rating-Agenturen eher noch gewachsen. Gerade die Finanzkrise des griechischen Staates zeigt die Reaktionshandlungen der Politik vor dem Hintergrund drohender Bonitätsabwertungen durch die großen Agenturen. Das ist ein Umstand, der die grundlegende Erklärungslogik des Buches stützt und die Lektüre dennoch empfehlenswert macht.

Alexander Leipold

\section{INTERNATIONALE BEZIEHUNGEN}

Mello, Patrick. Democratic Participation in Armed Conflict: Military Involvement in Kosovo, Afghanistan, and Iraq. Basingstoke. Palgrave (Palgrave Studies in International Relations) 2014. 280 Seiten. 87,99€.

Die Militärinterventionen seit dem Ende des Ost-West-Konflikts sind treffend als „wars of choice“ beschrieben worden. Unbeschadet bündnispolitischer und -solidarischer Erwägungen lassen sie im Gegensatz zur Territorialund Bündnisverteidigung jedem einzelnen Staat erhebliche Spielräume bei der Entscheidung, selbst einen militärischen Beitrag zu leisten - oder eben davon abzusehen. Die Forschung zum so genannten „Demokratischen Frieden“ hat uns dabei gelehrt, mit Unterschieden zwischen Demokratien und Autokratien zu rechnen, hat aber auch den Blick auf die auffälligen Differenzen zwischen liberalen Demokratien verstellt. Das ist schon deshalb bedauerlich, weil die Unterschiede zwischen Demokratien für Theorien des Konfliktverhaltens ebenso bedeutsam sind wie ihre Gemeinsamkeiten im Vergleich zu Autokratien.

Die bisherige Forschung spiegelt unterschiedliche methodische Vorlieben diesseits und jenseits des Atlantiks wider: In den USA arbeitende Kollegen haben das Konfliktverhalten von Demokratien bevorzugt quantitativ und am liebsten gleich über die letzten fünfzig oder hundert Jahre untersucht. In Europa beheimatete Autoren hingegen neigen zu Zweifeln, ob auf diese Weise generalisierbare Befunde gewonnen werden können, und legen daher lieber qualitative Studien zum Konfliktverhalten einzelner oder weniger Demokratien in einzelnen oder wenigen Konflikten vor.

Patrick Mellos Studie zeichnet sich dadurch aus, die Vorzüge quantitativer und qualitativer Zugänge miteinander $\mathrm{zu}$ verbinden. In drei Fallstudien zu den Militärinterventionen im Kosovo 1999, in Afghanistan 2001 und im Irak 2003 werden die Beteiligungen von über zwanzig liberalen Demokratien verglichen. Im Gegensatz zu einigen quantitativen Studien werden „wars of 
choice" somit nicht mit anderen Konfliktformen, beispielsweise Territorialkonflikten, vermengt. Die Methode der „fuzzy set Qualitative Comparative Analysis" erlaubt darüber hinaus, den unvermeidlichen Verlust an Information über einzelne Fälle zu minimieren, beispielsweise, indem die Konfliktbeteiligung nicht dichotomisiert wird, sondern nach unterschiedlichen Graden militärischer Beteiligung gefragt wird.

Die Auswahl der möglichen Erklärungsfaktoren lässt den Einfluss der Forschung zum Demokratischen Frieden erkennen. Bei allen drei Interventionen wird geprüft, ob eine dem Einsatz kritisch gegenüber stehende öffentliche Meinung mit militärischer Zurückhaltung einhergeht. Auch das institutionelle Kernargument des Demokratischen Friedens wird getestet, indem nach dem Einfluss eines parlamentarischen Vetorechts auf die Einsatzentscheidung gefragt wird. An dieser Stelle zeigt sich ein Vorzug der von Mello verwendeten Methodik: Die „Qualitative Comparative Analysis" legt nämlich nahe, öffentliche Meinung und demokratische Institutionen nicht nur getrennt voneinander $\mathrm{zu}$ betrachten, sondern deren Zusammenspiel in den Mittelpunkt zu rücken. Das führt zu der Hypothese, dass die Gleichzeitigkeit von ablehnender öffentlicher Meinung und einem mit Vetorecht ausgestattetem Parlament einen Militäreinsatz besonders unwahrscheinlich werden lässt.

Geprüft wird darüber hinaus auch der Einfluss von militärischer Macht, von der ideologischen Ausrichtung der Regierung und von möglichen, in der Verfassung festgeschriebenen Restriktionen. Unter die letztgenannte Variable fallen beispielweise das Neutralitätsgebot in der Schweiz und in Österreich oder das Verbot militärischer Gewalt außerhalb von Selbstverteidigung und kollektiver Sicherheit, das beispielsweise im Falle Deutschlands einen Einsatz ohne ein Mandat des UN-Sicherheitsrates ausschließt.

Angesichts der relativ hohen Zahl untersuchter Staaten kann es nicht überraschen, dass keiner der untersuchten Einflussfaktoren die Entscheidungen aller Staaten in allen drei Interventionen erklären kann. Für statistische Untersuchungen würde das kein Problem darstellen, solange nur der Gesamtzusammenhang, ceteris paribus, statistisch signifikant ist. Im Rahmen einer "Qualitative Comparative Analysis" bedeutet jedoch jeder abweichende Fall einen erheblichen Abstrich an der Eleganz der Befunde, denn Ausnahmen lassen sich nicht ohne weiteres in das Vokabular notwendiger und hinreichender Bedingungen übersetzen. Die Wahl der Methode hat somit auch den Preis, dass sich die Diskussion der Befunde oft sperrig liest und dem Leser der Wunsch nach einer einfachen „take home message" verwehrt bleibt. Positiv gewendet kann man allerdings feststellen, dass die Methodik zu einer nuancierten Interpretation der Daten zwingt, die der Komplexität liberaldemokratischer Entscheidungsprozesse entspricht.

Insgesamt beeindruckt das Buch durch die Genauigkeit und Gründlichkeit, mit der auf methodisch innovative Weise eine Reihe von Erklärungsfaktoren an einer großen Zahl von Fällen getestet wird. Gerade weil es auf einfache Botschaften verzichtet, leistet es einen wichtigen Beitrag zur Erforschung des Konfliktverhaltens von Demokratien und zur Außenpolitikanalyse im Allgemeinen.

Wolfgang Wagner 